American Journal of Environmental Sciences 5 (3): 406-412, 2009

ISSN 1553-345X

(C) 2009 Science Publications

\title{
Effects of Converting Secondary Forest to Oil Palm Plantation on Peat Soil Carbon and Nitrogen and other Soil Chemical Properties
}

\author{
${ }^{1}$ Makilan Muniandy, ${ }^{1}$ Osumanu Haruna Ahmed, ${ }^{1}$ Nik Muhamad Ab. Majid \\ and ${ }^{2}$ Mohd Khanif Yusop \\ ${ }^{1}$ Department of Crop Science, Faculty of Agriculture and Food Science, \\ University Putra Malaysia, Bintulu Campus, Sarawak, 97008 Bintulu, Sarawak, Malaysia \\ ${ }^{2}$ Department of Land Management, Faculty of Agriculture, \\ University Putra Malaysia, 43400 Serdang, Selangor, Malaysia
}

\begin{abstract}
Problem statement: Peatlands are natural sequesters of carbon and nitrogen. Once they are disturbed the tendency to lose carbon and nitrogen to the environment is very high. This study investigated the effect of converting peat land forest into oil palm plantation on soil chemical properties with particular emphasis on carbon and nitrogen storage. Approach: Soil samples were collected randomly at depths $0-25$ and $25-50 \mathrm{~cm}$ from a secondary forest and from four different ages of oil palm plantations at woodman oil palm plantation located in Sarawak, Malaysia. Soil pH in water and $\mathrm{KCl}$, Organic Matter (OM), Organic Carbon (OC), Total Nitrogen (TN), Organic Nitrogen (ON), ammonium, nitrate, available phosphorous, carbon to nitrogen ratio, carbon to phosphorous ratio and bulk density were determined using standard procedures. The bulk density method was used to quantify Carbon $(\mathrm{C})$, Nitrogen $(\mathrm{N})$, ammonium, nitrate and available phosphorous storage on per hectare basis. Results: Statistical analysis showed that the OC content was statistically similar for all soil depths and vegetation types (forest or plantation). The TN content was statistically higher for secondary forest. Conclusion: Regardless of depth, $\mathrm{C}$ sequestration was not altered due to land use change but the secondary forest had higher stores of soil $\mathrm{N}$.
\end{abstract}

Key words: Peat, oil palm plantation, secondary forest, organic C, total N, soil chemical properties

\section{INTRODUCTION}

Peat is defined as the accumulation of purely one hundred percent organic matter and the distinction between soil and vegetative accumulation is not clear ${ }^{[1]}$. The soil division of Sarawak, Malaysia adopted a more recent definition which is based on soil partition, i.e. soils that have $50 \mathrm{~cm}$ or more Soil Organic Matter (SOM) within $100 \mathrm{~cm}$ or more than twice that of mineral soil materials overlying bedrock within 50 $\mathrm{cm}^{[2]}$. Peat soils form generally in large basin swamps which are dome-shaped. Organic soil deposits occupying the central portion of the dome, generally known as 'ombrogenous peat', comprises mainly of disaggregated tree trunks, branches, leaves, roots and fruits.

Occurrence of peat soil around the globe is about 423.7 million ha. In Asia, there are about 24.9 million ha of peat soil and in Malaysia about 2.5 million ha of peat soil where the estimated percentage of SOM in tropical forest is $100^{[11]}$. The occurrence of peat soil in
Sarawak, Malaysia is estimated to be about 1.8 million ha which is the largest hectarage for any of the Malaysian states ${ }^{[3]}$.

Organic carbon and TN contents in peat soil are very high with the OC contents ranging from $12-60 \%^{[1]}$. The surface of peat soils contain more $\mathrm{C}$ compared to the subsoil with values such as $58 \%$ at the surface and $25 \%$ in the subsoil ${ }^{[4]}$ and typical of deep peat soils. Organic nitrogen in shallow peat soils can range from $0.5-2.1 \%$ and in the deep peat soils the values can range from $1.1-1.7 \%{ }^{[5]}$ with $\mathrm{N}$ levels in the deep peat being higher compared to shallow peat soils ${ }^{[1]}$.

High contents of $\mathrm{C}$ and $\mathrm{N}$ as well as other nutrients in peat soil have led to large hectares of peat land being developed for agricultural uses. Water holding capacity, cation exchange capability (CEC) and high moisture content in peat soils make them ideal for agriculture. Therefore, it is no surprise that in Sarawak, Malaysia around 554.8 thousand ha of peat land has been utilized out of the total 1.8 million ha of peat land ${ }^{[3]}$. The objective of this study was to compare the TN, OC

Corresponding Author: Osumanu Haruna Ahmed, Department of Crop Science, Faculty of Agriculture and Food Sciences, University Putra Malaysia, Bintulu Campus, Sarawak, Malaysia Tel: +6086855406 Fax: +608685415 
storage and other soil chemical properties of a secondary forest and young oil palm plantations that were previously secondary forest.

\section{MATERIALS AND METHODS}

The soil samples used in this study were taken from a secondary forest and Woodman oil palm plantation Kuala Tatau, Sarawak, Malaysia. The size of each experimental plot for the two locations was $30 \times 40 \mathrm{~m}$. The ages of the oil palm plantations available sampled were one, three, four and five years old. Ten soil samples were taken using a peat soil auger at 0-25 and $25-50 \mathrm{~cm}$ depths. The samples were air dried and then sieved to pass $2 \mathrm{~mm}$ sieve.

The coring method was used to determine the bulk density at these depths. The determination of soil $\mathrm{pH}$ was done by using a ratio of 1:2.5 soil to distilled water suspension ( $\mathrm{pH}$ in water) and $1 \mathrm{M}$ of $\mathrm{KCl}(\mathrm{pH}$ in $1 \mathrm{M}$ potassium chloride) using a glass electrode ${ }^{[7]}$. Ten grams of soil samples were weighed and placed in plastic vials to which $25 \mathrm{~mL}$ of distilled water was added, shaken and allowed to settle for $24 \mathrm{~h}$. The samples were again gently swirled and the $\mathrm{pH}$ determined using a glass electrode. The same was done using $25 \mathrm{~mL}$ of $1 \mathrm{~N} \mathrm{KCl}$ but the samples were allowed to equilibrate for $10 \mathrm{~min}$ before the $\mathrm{pH}$ measurement with a glass electrode.

SOM was determined by the wet oxidation using sulfuric acid and digestion catalyst $\left(\mathrm{K}_{2} \mathrm{SO}_{4}, \mathrm{CuSO}_{4}\right.$ and SeO: ratio 100:10:1). Soil TN was determined by the kjeldahl method ${ }^{[7]}$.

To determine the inorganic nitrogen content (ammonium and nitrate $\mathrm{N}$ ) in the peat, $0.5 \mathrm{~g}$ of soil sample was weighed and placed into a conical flask. A $50 \mathrm{~mL}$ of $2 \mathrm{M}$ of $\mathrm{KCl}$ solution was added in the conical flask and shaken for $1 \mathrm{~h}$ using a mechanical shaker. The mixture was filtered and the filtrate used for subsequent ammonium measurement through distillation and titration. Twenty $\mathrm{mL}$ of the filtrate was pippeted into a distillation flask and $0.2 \mathrm{~g}$ of $\mathrm{MgO}$ was added, followed by distillation. The gas produced was collected in a conical flask containing $10 \mathrm{~mL}$ of Boric acid + indicator solution. The resulting solution containing the gas was titrated using $0.01 \mathrm{M} \mathrm{HCl}$ solution. Nitrate content was determined using the same sample for ammonium determination. Exactly $0.2 \mathrm{~g}$ of Devarda alloy was added to the same distillation flask and the distillation process carried out. The gas produced was collected in a conical flask with $10 \mathrm{~mL}$ of Boric acid + indicator solution. The resulting solution containing the gas was titrated was titrated using $0.01 \mathrm{M} \mathrm{HCl}$ solution.
TOC was estimated using the Loss-On-Ignition (LOI) method ${ }^{[8]}$.

TOC and TON per hectare basis was then calculated using the bulk density method ${ }^{[9]}$. The ratio of percentage of the TOC and TON was used to determine the $\mathrm{C} / \mathrm{N}$ ratio of the soil.

Available $\mathrm{P}$ in the peat soil was measured by the colorimetric determination of $\mathrm{P}^{[11,12]}$.

Data was analyzed using the Statistical Analysis System (SAS) version 9.1 by means of ANOVA and means separation was done using the Tukey test. T-test was used to compare results at $0-25$ and $25-50 \mathrm{~cm}$ soil depths.

\section{RESULTS}

The soil pH of secondary forest and four year old oil palm plantation showed no significant differences regardless of depth (Table 1). The soil $\mathrm{pH}$ of the secondary forest and 1, 3 and 5 year old oil palm plantation at $0-25 \mathrm{~cm}$ were not significantly different but were so for $25-50 \mathrm{~cm}$. The soil $\mathrm{pH}$ at $0-25 \mathrm{~cm}$ for the 5 year old oil palm plantation was significantly higher than those of $1,3,4$ and secondary forest (Table 2) which was not the case for $25-50 \mathrm{~cm}$.

Vegetation type (oil palm or forest), oil palm age and soil depth had no significant effect on soil bulk density (Table 3 and 4). However, the bulk density values were typical of tropical peat bulk density.

Organic matter content decreased significantly with soil depth between secondary forest and oil palms aged 4 but no significant differences between oil palm ages 1, 3 and 5 (Table 5). Depth of sampling did not show any significant effect on soil organic matter content (Table 6).

Table 1: Soil $\mathrm{pH}$ of secondary forest and different ages of oil palm plantation

\begin{tabular}{ll}
\hline Location $(\mathrm{cm})$ & $\mathrm{pH}$ (Water) \\
\hline Secondary forest: & $3.321^{\mathrm{a}} \pm 0.029$ \\
$0-25$ & $3.372^{\mathrm{a}} \pm 0.034$ \\
25-50 & $3.371^{\mathrm{a}} \pm 0.033$ \\
1 Year old oil palm plantation: & $3.745^{\mathrm{b}} \pm 0.044$ \\
$0-25$ & \\
$25-50$ & $3.315^{\mathrm{a}} \pm 0.066$ \\
3 Year old oil palm plantation: & $3.631^{\mathrm{b}} \pm 0.115$ \\
$0-25$ & \\
25-50 & $3.105^{\mathrm{a}} \pm 0.073$ \\
4 Year old oil palm plantation: & $3.102^{\mathrm{a}} \pm 0.049$ \\
$0-25$ & \\
25-50 & $3.633^{\mathrm{a}} \pm 0.097$ \\
5 Year old oil palm plantation: & $3.394^{\mathrm{b}} \pm 0.050$ \\
$0-25$ &
\end{tabular}

Note: Means within column with same alphabets are not significantly different at $\mathrm{p}=0.05 \mathrm{using}$ dependent $\mathrm{t}$-test 
Am. J. Environ. Sci., 5 (3): 406-412, 2009

Table 2: Soil $\mathrm{pH}$ of secondary forest and different ages of oil palm plantation

\begin{tabular}{ll}
\hline Depth of sample $\mathbf{( 0 - 2 5} \mathbf{~ c m})$ & $\mathrm{pH}($ water) \\
\hline Secondary forest & $3.321^{\mathrm{bc}}$ \\
1 Year old oil palm plantation & $3.371^{\mathrm{b}}$ \\
3 Year old oil palm plantation & $3.315^{\mathrm{bc}}$ \\
4 Year old oil palm plantation & $3.105^{\mathrm{c}}$ \\
5 Year old oil palm plantation & $3.633^{\mathrm{a}}$ \\
Depth of sample (25-50 cm): & \\
Secondary forest & $3.372^{\mathrm{b}}$ \\
1 Year old oil palm plantation & $3.745^{\mathrm{a}}$ \\
3 Year old oil palm plantation & $3.631^{\mathrm{b}}$ \\
4 Year old oil palm plantation & $3.102^{\mathrm{c}}$ \\
5 Year old oil palm plantation & $3.394^{\mathrm{b}}$ \\
\hline Note: Means within column with same alphabets are not significantly \\
different at p = 0.05 using Tukey's test
\end{tabular}

Table 3: Bulk densities of secondary forest and different ages of oil palm plantation

\begin{tabular}{ll}
\hline Location $(\mathrm{cm})$ & Bulk density $\left(\mathrm{g} \mathrm{cm}^{-3}\right)$ \\
\hline Secondary forest: & $0.300^{\mathrm{a}} \pm 0.007$ \\
$0-25$ & $0.275^{\mathrm{a}} \pm 0.005$ \\
25-50 & $0.298^{\mathrm{a}} \pm 0.004$ \\
1 Year old oil palm plantation: & $0.293^{\mathrm{a}} \pm 0.007$ \\
$0-25$ & \\
25-50 & $0.299^{\mathrm{a}} \pm 0.007$ \\
3 Year old oil palm plantation: & $0.304^{\mathrm{a}} \pm 0.007$ \\
0-25 & \\
25-50 & $0.310^{\mathrm{a}} \pm 0.006$ \\
4 Year old oil palm plantation: & $0.293^{\mathrm{a}} \pm 0.008$ \\
0-25 & \\
25-50 & $0.289^{\mathrm{a}} \pm 0.003$ \\
5 Year old oil palm plantation: & $0.284^{\mathrm{a}} \pm 0.004$ \\
0-25 &
\end{tabular}

Note: Means with same alphabet are not significantly different at $\mathrm{p}=0.05$ using dependent t-test

Table 4: Bulk densities of secondary forest and different ages of oil palm plantation

\begin{tabular}{ll}
\hline Depth of sample $\mathbf{( 0 - 2 5} \mathbf{~ c m})$ & Bulk density $\left(\mathrm{g} \mathrm{cm}^{-3}\right)$ \\
\hline Secondary forest & $0.300^{\mathrm{a}}$ \\
1 Year old oil palm plantation & $0.298^{\mathrm{a}}$ \\
3 Year old oil palm plantation & $0.299^{\mathrm{a}}$ \\
4 Year old oil palm plantation & $0.310^{\mathrm{a}}$ \\
5 Year old oil palm plantation & $0.289^{\mathrm{a}}$ \\
Depth of sample $(\mathbf{2 5 - 5 0} \mathbf{~ c m}):$ & \\
Secondary forest & $0.275^{\mathrm{a}}$ \\
1 Year old oil palm plantation & $0.293^{\mathrm{ab}}$ \\
3 Year old oil palm plantation & $0.304^{\mathrm{b}}$ \\
4 Year old oil palm plantation & $0.293^{\mathrm{ab}}$ \\
5 Year old oil palm plantation & $0.284^{\mathrm{ab}}$ \\
\hline
\end{tabular}

Note: Means with same alphabet are not significantly different at $\mathrm{p}=0.05$ using Tukey's test

There were significant differences in soil carbon contents for the two depths for secondary forest, 4 and 5 year old oil palm plantation but not for the 1 and 3 year old plantations. However, the amount of carbon per hectare was neither affected by depth, vegetation type and age of oil palm plantation (Table 7 and 8).
Table 5: Organic matter contents of secondary forest and different ages of oil palm plantation

\begin{tabular}{ll}
\hline Location $(\mathrm{cm})$ & $\begin{array}{l}\text { Organic } \\
\text { matter }(\%)\end{array}$ \\
\hline Secondary forest: & \\
$0-25$ & $95.2300^{\mathrm{a}} \pm 0.536$ \\
$25-50$ & $96.6020^{\mathrm{b}} \pm 0.326$ \\
1 Year old oil palm plantation: & \\
$0-25$ & $89.9520^{\mathrm{a}} \pm 3.570$ \\
$25-50$ & $100.745^{\mathrm{a}} \pm 3.429$ \\
3 Year old oil palm plantation: & \\
$0-25$ & $96.6140^{\mathrm{a}} \pm 0.420$ \\
25-50 & $93.1360^{\mathrm{a}} \pm 3.948$ \\
4 Year old oil palm plantation: & $85.4940^{\mathrm{a}} \pm 1.334$ \\
$0-25$ & $92.3340^{\mathrm{b}} \pm 0.928$ \\
25-50 & $94.2320^{\mathrm{a}} \pm 1.508$ \\
5 Year old oil palm plantation: & $92.8500^{\mathrm{a}} \pm 1.665$ \\
$0-25$ &
\end{tabular}

Note: Means with same alphabet a re not significantly different at $\mathrm{p}=0.05$ using dependent $\mathrm{t}$-test

Table 6: Organic matter contents of secondary forest and different ages of oil palm plantation

\begin{tabular}{lc}
\hline Depth of sample (0-25 cm) & Organic matter (\%) \\
\hline Secondary forest & $95.230^{\mathrm{a}}$ \\
1 Year old oil palm plantation & $89.952^{\mathrm{ab}}$ \\
3 Year old oil palm plantation & $96.614^{\mathrm{a}}$ \\
4 Year old oil palm plantation & $85.494^{\mathrm{b}}$ \\
5 Year old oil palm plantation & $94.232^{\mathrm{a}}$ \\
Depth of sample $(\mathbf{2 5 - 5 0} \mathbf{~ c m ) : ~}$ & \\
Secondary forest & $96.602^{\mathrm{a}}$ \\
1 Year old oil palm plantation & $100.745^{\mathrm{a}}$ \\
3 Year old oil palm plantation & $93.136^{\mathrm{a}}$ \\
4 Year old oil palm plantation & $92.334^{\mathrm{a}}$ \\
5 Year old oil palm plantation & $92.850^{\mathrm{a}}$
\end{tabular}

Note: Means with same alphabet are not significantly different at $\mathrm{p}=0.05$ using Tukey's test

Table 7: Organic carbon contents of secondary forest and different ages of oil palm plantation

\begin{tabular}{llc}
\hline Location $(\mathrm{cm})$ & $\begin{array}{l}\text { Organic } \\
\text { carbon }(\%)\end{array}$ & $\begin{array}{c}\text { Organic carbon } \\
\left(\mathrm{Mg} \mathrm{Ha}^{-1}\right)\end{array}$ \\
\hline Secondary forest: & $47.615^{\mathrm{a}} \pm 0.268$ & $356.604^{\mathrm{a}} \pm 8.095$ \\
$0-25$ & $48.301^{\mathrm{b}} \pm 0.163$ & $332.168^{\mathrm{a}} \pm 6.596$ \\
25-50 & $44.976^{\mathrm{a}} \pm 1.785$ & $334.528^{\mathrm{a}} \pm 13.101$ \\
1 Year old oil palm plantation: & $50.374^{\mathrm{a}} \pm 1.714$ & $368.988^{\mathrm{a}} \pm 18.255$ \\
$0-25$ & & \\
25-50 & $48.307^{\mathrm{a}} \pm 0.210$ & $361.412^{\mathrm{a}} \pm 9.878$ \\
3 Year old oil palm plantation: & $46.568^{\mathrm{a}} \pm 1.974$ & $353.686^{\mathrm{a}} \pm 17.010$ \\
$0-25$ & & \\
25-50 & $42.747^{\mathrm{a}} \pm 0.667$ & $330.932^{\mathrm{a}} \pm 7.920$ \\
4 Year old oil palm plantation: & $46.187^{\mathrm{b} \pm 0.470}$ & $338.676^{\mathrm{a}} \pm 8.378$ \\
0-25 & & \\
25-50 & $47.098^{\mathrm{a}} \pm 0.769$ & $340.876^{\mathrm{a}} \pm 8.878$ \\
5 Year old oil palm plantation: & $46.425^{\mathrm{b}} \pm 0.833$ & $329.904^{\mathrm{a}} \pm 5.397$ \\
$0-25$ & &
\end{tabular}

Note: Means within a column with the same alphabet are not significantly different at $\mathrm{p}=0.05 \mathrm{using}$ dependent t-test

In general, soil depth did not affect the amount of $\mathrm{N}$ and the quantity of $\mathrm{N}$ per hectare (Table 9 ). However, these variables were significantly higher for the secondary forest than the oil palm ages of 1, 3, 4 and 5 (Table 10). 
Am. J. Environ. Sci., 5 (3): 406-412, 2009

Table 8: Organic carbon contents of secondary forest and different ages of oil palm plantation

\begin{tabular}{lll}
\hline Depth of sample $\mathbf{( 0 - 2 5 ~ c m )}$ & $\begin{array}{l}\text { Organic } \\
\text { carbon }(\%)\end{array}$ & $\begin{array}{l}\text { Organic carbon } \\
\left(\mathrm{Mg} \mathrm{Ha}^{-1}\right)\end{array}$ \\
\hline Secondary forest & $47.615^{\mathrm{a}}$ & $356.604^{\mathrm{a}}$ \\
1 Year old oil palm plantation & $44.976^{\mathrm{ab}}$ & $334.528^{\mathrm{a}}$ \\
3 Year old oil palm plantation & $48.307^{\mathrm{a}}$ & $361.412^{\mathrm{a}}$ \\
4 Year old oil palm plantation & $42.747^{\mathrm{b}}$ & $330.932^{\mathrm{a}}$ \\
5 Year old oil palm plantation & $47.098^{\mathrm{a}}$ & $340.876^{\mathrm{a}}$ \\
Depth of sample (25-50 cm): & & \\
Secondary forest & $48.301^{\mathrm{a}}$ & $332.168^{\mathrm{a}}$ \\
1 Year old oil palm plantation & $50.374^{\mathrm{a}}$ & $368.988^{\mathrm{a}}$ \\
3 Year old oil palm plantation & $46.568^{\mathrm{a}}$ & $353.686^{\mathrm{a}}$ \\
4 Year old oil palm plantation & $46.187^{\mathrm{a}}$ & $338.676^{\mathrm{a}}$ \\
5 Year old oil palm plantation & $46.425^{\mathrm{a}}$ & $329.904^{\mathrm{a}}$ \\
\hline N
\end{tabular}

Note: Means with same alphabet are not significantly different at $\mathrm{p}=0.05$ using Tukey's test

Table 9: Total nitrogen contents of secondary forest and different ages of oil palm plantation

\begin{tabular}{|c|c|c|}
\hline Location (cm) & \multicolumn{2}{|r|}{$\begin{array}{l}\text { Total nitrogen } \\
\text { b) }\left(\mathrm{Mg} \mathrm{Ha}^{-1}\right)\end{array}$} \\
\hline \multicolumn{3}{|c|}{ Secondary forest: } \\
\hline $0-25$ & $2.401^{\mathrm{a}} \pm 0.175$ & $17.96^{\mathrm{a}} \pm 1.743$ \\
\hline $25-50$ & $2.468^{\mathrm{a}} \pm 0.132$ & $17.00^{\mathrm{a}} \pm 1.057$ \\
\hline \multicolumn{3}{|c|}{1 Year old oil palm plantation: } \\
\hline $0-25$ & $2.328^{\mathrm{a}} \pm 0.289$ & $17.31^{\mathrm{a}} \pm 2.409$ \\
\hline $25-50$ & $1.537^{\mathrm{a}} \pm 0.182$ & $11.28^{\mathrm{a}} \pm 1.684$ \\
\hline \multicolumn{3}{|c|}{3 Year old oil palm plantation: } \\
\hline $0-25$ & $1.304^{\mathrm{a}} \pm 0.092$ & $9.790^{\mathrm{a}} \pm 0.830$ \\
\hline $25-50$ & $1.073^{\mathrm{b}} \pm 0.035$ & $7.909^{\mathrm{a}} \pm 0.533$ \\
\hline \multicolumn{3}{|c|}{4 Year old oil palm plantation: } \\
\hline $0-25$ & $1.375^{\mathrm{a}} \pm 0.116$ & $10.64^{\mathrm{a}} \pm 0.533$ \\
\hline $25-50$ & $1.489^{\mathrm{a}} \pm 0.294$ & $10.86^{\mathrm{a}} \pm 2.052$ \\
\hline \multicolumn{3}{|c|}{5 Year old oil palm plantation: } \\
\hline $0-25$ & $1.369^{\mathrm{a}} \pm 0.060$ & $9.910^{\mathrm{a}} \pm 0.559$ \\
\hline $25-50$ & $1.120^{\mathrm{a}} \pm 0.139$ & $7.940^{\mathrm{a}} \pm 0.878$ \\
\hline
\end{tabular}

Note: Means with same alphabet are not significantly different at $\mathrm{p}=0.05$ using dependent $\mathrm{t}$-test

Table 10: Total nitrogen contents of secondary forest and different ages of oil palm plantation

\begin{tabular}{llc}
\hline Depth of sample $\mathbf{( 0 - 2 5 ~ c m )}$ & $\begin{array}{l}\text { Total } \\
\text { nitrogen }(\%)\end{array}$ & $\begin{array}{l}\text { Total nitrogen } \\
\left(\mathrm{Mg} \mathrm{Ha}^{-1}\right)\end{array}$ \\
\hline Secondary forest & $2.401^{\mathrm{a}}$ & $17.96^{\mathrm{a}}$ \\
1 Year old oil palm plantation & $2.328^{\mathrm{a}}$ & $17.31^{\mathrm{a}}$ \\
3 Year old oil palm plantation & $1.304^{\mathrm{b}}$ & $9.79^{\mathrm{b}}$ \\
4 Year old oil palm plantation & $1.375^{\mathrm{b}}$ & $10.64^{\mathrm{b}}$ \\
5 Year old oil palm plantation & $1.369^{\mathrm{b}}$ & $9.91^{\mathrm{b}}$ \\
Depth of sample (25-50 cm): & & \\
Secondary forest & $2.468^{\mathrm{a}}$ & $17.00^{\mathrm{a}}$ \\
1 Year old oil palm plantation & $1.537^{\mathrm{b}}$ & $11.28^{\mathrm{ab}}$ \\
3 Year old oil palm plantation & $1.073^{\mathrm{b}}$ & $7.99^{\mathrm{b}}$ \\
4 Year old oil palm plantation & $1.489^{\mathrm{b}}$ & $10.86^{\mathrm{b}}$ \\
5 Year old oil palm plantation & $1.120^{\mathrm{b}}$ & $7.94^{\mathrm{b}}$ \\
\hline
\end{tabular}

Note: Means with same alphabet are not significantly different at $\mathrm{p}=0.05$ using Tukey's test

Regardless of depth, exchangeable ammonium concentration was not statistically different (Table 11) for 1,3 and 4 year old plantations but there was significant increase with increasing depth for secondary forest and 5 year old oil palm plantation. However,
Table 11: Ammonium contents of secondary forest and different ages of oil palm plantation

\begin{tabular}{lcl}
\hline Location $(\mathrm{cm})$ & Ammonium $(\%)$ & $\begin{array}{l}\text { Ammonium } \\
\left(\mathrm{Mg} \mathrm{Ha}^{-1}\right)\end{array}$ \\
\hline $\begin{array}{l}\text { Secondary forest } \\
0-25\end{array}$ & $0.043^{\mathrm{a}} \pm 0.003$ & $0.329^{\mathrm{a}} \pm 0.026$ \\
$25-50$ & $0.060^{\mathrm{b}} \pm 0.004$ & $0.411^{\mathrm{b}} \pm 0.024$ \\
1 Year old oil palm plantation & & \\
$0-25$ & $0.198^{\mathrm{a}} \pm 0.004$ & $1.474^{\mathrm{a}} \pm 0.046$ \\
25-50 & $0.199^{\mathrm{a}} \pm 0.006$ & $1.452^{\mathrm{a}} \pm 0.048$ \\
3 Year old oil palm plantation & & \\
$0-25$ & $0.163^{\mathrm{a}} \pm 0.011$ & $1.215^{\mathrm{a}} \pm 0.067$ \\
25-50 & $0.156^{\mathrm{a}} \pm 0.008$ & $1.185^{\mathrm{a}} \pm 0.090$ \\
4 Year old oil palm plantation & & \\
$0-25$ & $0.152^{\mathrm{a}} \pm 0.002$ & $1.177^{\mathrm{a}} \pm 0.032$ \\
25-50 & $0.155^{\mathrm{a}} \pm 0.003$ & $1.135^{\mathrm{a}} \pm 0.022$ \\
5 Year old oil palm plantation & & \\
$0-25$ & $0.240^{\mathrm{a}} \pm 0.003$ & $1.736^{\mathrm{a}} \pm 0.034$ \\
25-50 & $0.255^{\mathrm{b}} \pm 0.003$ & $1.812^{\mathrm{a}} \pm 0.022$ \\
\hline
\end{tabular}

Note: Means with same alphabet are not significantly different at $\mathrm{p}=0.05$ using dependent $\mathrm{t}$-test

Table 12: Ammonium contents of secondary forest and different ages of oil palm plantation

\begin{tabular}{lll}
\hline Depth of sample (0-25 cm) & Ammonium (\%) & $\begin{array}{l}\text { Ammonium } \\
\left(\mathrm{Mg} \mathrm{Ha}^{-1}\right)\end{array}$ \\
\hline Secondary forest & $0.043^{\mathrm{a}}$ & $0.329^{\mathrm{a}}$ \\
1 Year old oil palm plantation & $0.198^{\mathrm{b}}$ & $1.472^{\mathrm{b}}$ \\
3 Year old oil palm plantation & $0.163^{\mathrm{c}}$ & $1.215^{\mathrm{c}}$ \\
4 Year old oil palm plantation & $0.152^{\mathrm{c}}$ & $1.177^{\mathrm{c}}$ \\
5 Year old oil palm plantation & $0.240^{\mathrm{d}}$ & $1.736^{\mathrm{d}}$ \\
Depth of sample (25-50 cm): & & \\
Secondary forest & $0.060^{\mathrm{a}}$ & $0.411^{\mathrm{a}}$ \\
1 Year old oil palm plantation & $0.199^{\mathrm{b}}$ & $1.452^{\mathrm{b}}$ \\
3 Year old oil palm plantation & $0.156^{\mathrm{c}}$ & $1.185^{\mathrm{c}}$ \\
4 Year old oil palm plantation & $0.155^{\mathrm{c}}$ & $1.135^{\mathrm{c}}$ \\
5 Year old oil palm plantation & $0.255^{\mathrm{d}}$ & $1.812^{\mathrm{d}}$ \\
\hline
\end{tabular}

Note: Mean with same alphabet are not significantly different at $\mathrm{p}=0.05$ using Tukey's test

except for the secondary forest which showed decrease in ammonium $\mathrm{N}$ per hectare with increasing depth, there were no significant differences in ammonium $\mathrm{N}$ per hectare for the four different ages of oil palm plantation at both soil depths.

Comparing the ammonium $\mathrm{N}$ content and the amount per hectare for secondary forest for the four different ages of oil palm plantation indicate that 1, 3, 4 and 5 year plantations had significantly higher quantities regardless of depth (Table 12). A similar observation was made for nitrate $\mathrm{N}$ (Table 13).

Available $\mathrm{P}$ decreased with increasing depth (Table 14) and the amount per hectare for the four oil palm plantations had significantly higher amount compared to the secondary forest at $25-50 \mathrm{c} \mathrm{cm}$ depth (Table 15). 
Am. J. Environ. Sci., 5 (3): 406-412, 2009

Table 13: Nitrate contents of secondary forest and different ages of oil palm plantation

\begin{tabular}{lll}
\hline Depth of sample $(\mathbf{0 - 2 5} \mathbf{~ c m})$ & Nitrate $(\%)$ & Nitrate $\left(\mathrm{Mg} \mathrm{Ha}^{-1}\right)$ \\
\hline Secondary forest & $0.041^{\mathrm{a}}$ & $0.306^{\mathrm{a}}$ \\
1 Year old oil palm plantation & $0.206^{\mathrm{b}}$ & $1.531^{\mathrm{b}}$ \\
3 Year old oil palm plantation & $0.146^{\mathrm{c}}$ & $1.093^{\mathrm{c}}$ \\
4 Year old oil palm plantation & $0.153^{\mathrm{c}}$ & $1.186^{\mathrm{c}}$ \\
5 Year old oil palm plantation & $0.237^{\mathrm{d}}$ & $1.715^{\mathrm{b}}$ \\
Depth of sample $(\mathbf{2 5 - 5 0} \mathbf{~ c m}):$ & & \\
Secondary forest & $0.054^{\mathrm{a}}$ & $0.374^{\mathrm{a}}$ \\
1 Year old oil palm plantation & $0.208^{\mathrm{b}}$ & $1.520^{\mathrm{b}}$ \\
3 Year old oil palm plantation & $0.150^{\mathrm{c}}$ & $1.140^{\mathrm{c}}$ \\
4 Year old oil palm plantation & $0.151^{\mathrm{c}}$ & $1.106^{\mathrm{c}}$ \\
5 Year old oil palm plantation & $0.239^{\mathrm{d}}$ & $1.701^{\mathrm{d}}$ \\
\hline
\end{tabular}

Note: Means with same alphabet are not significantly different at $\mathrm{p}=$ 0.05 using Tukey's test

Table 14: Available P contents of secondary forest and different ages of oil palm plantation

\begin{tabular}{|c|c|c|}
\hline Location (cm) & Available P (\%) & $\begin{array}{l}\text { Available P } \\
\quad\left(\mathrm{Mg} \mathrm{Ha}^{-1}\right)\end{array}$ \\
\hline \multicolumn{3}{|c|}{ Secondary forest: } \\
\hline $0-25$ & $0.0047^{\mathrm{a}} \pm 0.00020$ & $0.0355^{\mathrm{a}} \pm 0.0023$ \\
\hline $25-50$ & $0.0024^{\mathrm{b}} \pm 0.00004$ & $0.0168^{\mathrm{b}} \pm 0.0005$ \\
\hline \multicolumn{3}{|c|}{1 Year old oil palm plantation: } \\
\hline $0-25$ & $0.0043^{\mathrm{a}} \pm 0.00024$ & $0.0321^{\mathrm{a}} \pm 0.0017$ \\
\hline $25-50$ & $0.0018^{\mathrm{b}} \pm 0.00009$ & $0.0128^{\mathrm{b}} \pm 0.0005$ \\
\hline \multicolumn{3}{|c|}{3 Year old oil palm plantation: } \\
\hline $0-25$ & $0.0027^{\mathrm{a}} \pm 0.00029$ & $0.0204^{\mathrm{a}} \pm 0.0020$ \\
\hline $25-50$ & $0.0013^{\mathrm{b}} \pm 0.00004$ & $0.0099^{\mathrm{b}} \pm 0.0005$ \\
\hline \multicolumn{3}{|c|}{4 Year old oil palm plantation: } \\
\hline $0-25$ & $0.0021^{\mathrm{a}} \pm 0.00014$ & $0.0159^{\mathrm{a}} \pm 0.0010$ \\
\hline $25-50$ & $0.0011^{\mathrm{b}} \pm 0.00007$ & $0.0078^{\mathrm{b}} \pm 0.0007$ \\
\hline \multicolumn{3}{|c|}{5 Year old oil palm plantation: } \\
\hline $0-25$ & $0.0036^{\mathrm{a}} \pm 0.00035$ & $0.0264^{\mathrm{a}} \pm 0.0026$ \\
\hline $25-50$ & $0.0016^{\mathrm{b}} \pm 0.00009$ & $0.0116^{\mathrm{b}} \pm 0.0008$ \\
\hline
\end{tabular}

Note: Means with same alphabet are not significantly different at $\mathrm{p}=0.05$ using dependent t-test

Table 15: Available $\mathrm{P}$ contents of secondary forest and different ages of oil palm plantation

\begin{tabular}{lll}
\hline Depth of sample (0-25 cm) & Available P (\%) & $\begin{array}{l}\text { Available P } \\
(\mathrm{Mg} / \mathrm{Ha})\end{array}$ \\
\hline Secondary forest & $0.0047^{\mathrm{a}}$ & $0.0355^{\mathrm{a}}$ \\
1 Year old oil palm plantation & $0.0043^{\mathrm{a}}$ & $0.0321^{\mathrm{ab}}$ \\
3 Year old oil palm plantation & $0.0027^{\mathrm{bc}}$ & $0.0204^{\mathrm{cd}}$ \\
4 Year old oil palm plantation & $0.0021^{\mathrm{c}}$ & $0.0159^{\mathrm{d}}$ \\
5 Year old oil palm plantation & $0.0036^{\mathrm{ab}}$ & $0.0264^{\mathrm{bc}}$ \\
Depth of sample (25-50 cm): & & \\
Secondary forest & $0.0024^{\mathrm{a}}$ & $0.0168^{\mathrm{a}}$ \\
1 Year old oil palm plantation & $0.0018^{\mathrm{b}}$ & $0.0128^{\mathrm{b}}$ \\
3 Year old oil palm plantation & $0.0013^{\mathrm{c}}$ & $0.0099^{\mathrm{cd}}$ \\
4 Year old oil palm plantation & $0.0011^{\mathrm{c}}$ & $0.0078^{\mathrm{d}}$ \\
5 Year old oil palm plantation & $0.0016^{\mathrm{b}}$ & $0.0116^{\mathrm{bc}}$ \\
\hline Ner Malion
\end{tabular}

Note: Means with same alphabet are not significantly different at $\mathrm{p}=0.05$ using Tukey's test

The $\mathrm{C} / \mathrm{N}$ ratio was not statistically different regardless of depth (Table 16) but it was generally lower for the secondary forest compared with the different oil palm ages especially for $0-25 \mathrm{~cm}$ soil depth (Table 17).
Table 16: Carbon to nitrogen ratio for secondary forest and different ages of oil palm plantation

\begin{tabular}{ll}
\hline Location $(\mathrm{cm})$ & $\mathrm{C}: \mathrm{N}$ \\
\hline Secondary forest & \\
$0-25$ & $20.822^{\mathrm{a}} \pm 1.566$ \\
$25-50$ & $20.045^{\mathrm{a}} \pm 0.990$ \\
1 Year old oil palm plantation & $22.127^{\mathrm{a}} \pm 2.930$ \\
$0-25$ & $40.904^{\mathrm{a}} \pm 8.352$ \\
$25-50$ & \\
3 Year old oil palm plantation & $38.641^{\mathrm{a}} \pm 2.569$ \\
$0-25$ & $43.802^{\mathrm{a}} \pm 2.936$ \\
$25-50$ & \\
4 Year old oil palm plantation & $32.968^{\mathrm{a}} \pm 2.533$ \\
$0-25$ & $36.178^{\mathrm{a}} \pm 3.027$ \\
$25-50$ & \\
5 Year old oil palm plantation & $34.841^{\mathrm{a}} \pm 1.202$ \\
$0-25$ & $47.993^{\mathrm{a}} \pm 6.589$ \\
$25-50$ &
\end{tabular}

Note: Means with same alphabet are not significantly different at $\mathrm{p}=0.05$ using dependent $\mathrm{t}$-test

Table 17: Carbon to nitrogen ratio for secondary forest and different ages of oil palm plantation

Depth of sample $(0-25 \mathrm{~cm})$

\begin{tabular}{ll} 
& $\mathrm{C}: \mathrm{N}$ \\
\hline Secondary forest & $20.822^{\mathrm{a}}$ \\
1 Year old oil palm plantation & $22.127^{\mathrm{a}}$ \\
3 Year old oil palm plantation & $38.641^{\mathrm{b}}$ \\
4 Year old oil palm plantation & $32.968^{\mathrm{b}}$ \\
5 Year old oil palm plantation & $34.841^{\mathrm{b}}$ \\
Depth of sample (25-50 cm): & \\
Secondary forest & $20.045^{\mathrm{a}}$ \\
1 Year old oil palm plantation & $40.904^{\mathrm{ab}}$ \\
3 Year old oil palm plantation & $43.802^{\mathrm{b}}$ \\
4 Year old oil palm plantation & $36.178^{\mathrm{ab}}$ \\
5 Year old oil palm plantation & $47.993^{\mathrm{b}}$ \\
\hline
\end{tabular}

Note: Means with same letters are not significantly different at $\mathrm{p}=0.05$ using Tukey's test

\section{DISCUSSION}

The $\mathrm{pH}$ values of this study were typical of tropical peat soils ${ }^{[3]}$. This may be attributed to water table management which is common in utilization of peat land soil. Lower water table causes microbes to decompose peat under aerobic conditions compared to anaerobic conditions in natural and undisturbed peat.

Previous studies by Andriesse ${ }^{[1]}$, Driessen and Suhardjo ${ }^{[12]}$ and Tie and $\mathrm{Kueh}^{[13]}$ reported bulk density values of $0.2 \mathrm{~g} \mathrm{~cm}^{-3}$ and lower. In this study, the bulk density was $0.3 \mathrm{~g} \mathrm{~cm}^{-3}$ which may be the result of mechanization and management practices done by the plantations. Compaction of the peat before planting is a common practice by plantations. For instance, heavy duty machinery is used during land clearing and drainage construction. Frequent machine traffic during fertilization and harvesting also compact the peat.

Percentage $\mathrm{OM}$ content in peat showed no significant differences within the sampling depth and 
among the ages of oil palm plantations. Tie ${ }^{[13]}$ stated that organic soil must at least contain $65 \%$ of organic matter. The observations in this study showed that organic matter content is as high as $80 \%$.

There was no significant difference in percentage organic carbon content between the secondary forest and the oil palm plantations for both sampling depths. However, for between depth comparisons, the secondary forest, four and five year old oil palm plantations showed significant differences. The values ranged from $40-50 \%$, this is consequent with the results obtained by Andriesse ${ }^{[1]}$ and Kanapathy ${ }^{[4]}$. There were no significant differences within depths and among locations of sampling for secondary forest and ages of oil palm plantations for organic carbon content in peat soil by weight. This is comparable to the values obtained for percentage organic carbon content.

The total $\mathrm{N}$ contents were consistent with those reported by $\mathrm{Tie}^{[13]}$ and higher total nitrogen content in secondary forest peat soil could be related to the genesis or the origin of the parent materials and the water management. For the oil palm plantation was much drained compared to the secondary forest. One, three, four and five year old oil palm plantations were significantly lower than the secondary forest because of initial land clearance, water table management, fertilizer application and liming. These practices tend to increase peat soil $\mathrm{pH}$ and boost the microbial activity which would degrade or mineralize the nitrogen in peat.

Percentage ammonium content and weight per hectare was lowest in secondary forest for both $0-25$ and $25-50 \mathrm{~cm}$ depths. This shows that inorganic form of nitrogen content in secondary forest is very low and does not affect the total nitrogen content much. The higher content of ammonium for the different ages of oil palm plantations could be due to $\mathrm{N}$ fertilizer inputs.

The insignificant differences in nitrate regardless of soil depth, forest type and oil palm plantation age could be due to leaching which is known to be high in tropical peat soils.

Available P content in both topsoil and subsoil was significantly higher compared to different ages of oil palm plantation. This is because of artificial low water table (managed) in oil palm plantation, which causes leaching during high rainfall that is prevalent in the tropics such as Malaysia.

The secondary forest showed lower mean $\mathrm{C} / \mathrm{N}$ ratio due to higher water table level which causes a condition wherein microbes need longer time to decompose the carbon and nitrogen. The different ages of oil palm plantation had higher $\mathrm{C} / \mathrm{N}$ ratio due to breakdown of nitrogen by microbes.

\section{CONCLUSION}

Regardless of depth, carbon storage was statistically similar to forest type and different ages of oil palm plantation, but that of nitrogen was significantly higher in the secondary forest. Studies on plantations much older than five years (for example 15-20 years and over) is worth doing as this could result in more clear differences in results emanating from the prolonged land use change and management.

\section{ACKNOWLEDGEMENT}

The researchers acknowledge the financial support and other services of University Putra Malaysia.

\section{REFERENCES}

1. Andriesse, J.P., 1988. Nature and Management of Tropical Peats. FAO Soils Bulletin 59. FAO, Rome. ISBN: 92-5-102657-2, pp: 165.

2. Tay, T.H., 1969. The distribution, characteristics, uses and potential of peat in West Malaysia. J. Trop. Geograph., 29: 58-63.

4. Kanapathy, K., 1976. Fertilizer requirement on peat soils. Malaysian Agric. J., 50: 292-307 http://orton.catie.ac.cr/cgi-

bin/wxis.exe/?IsisScript=CATALCO $\cdot$ xis\&method=po st\&formato $=2 \&$ cantidad $=1$ \&expresion $=m f n=046312$

5. Tie, Y.L. and J.S. Lim, 1992. Characteristic and classification of organic soils in Malaysia. In tropical peat. Proceedings of the International Symposium on Tropical Peatland, May 6-10, Malaysia Agricultural Research and Development Institute, Kuching, Sarawak, Malaysia, pp: 441. http://www.abebooks.co.uk/9789679361278/Tropi cal-Peat-Proceedings-International-Symposium9679361276/plp

6. Bremmer, J.M. and C.S. Sulvaney, 1982. Total Nitrogen. In: Methods of Soil Analysis, Part 2. A.L. et al., (Eds.). 2nd Edn., Agron. Mongr. 9. ASA and SSSA, Madison, WI., ISBN: 0891180729, pp: 595-624.

8. Ben-Dor, E. and A. Banin, 1989. Determination of organic matter content in arid-zone soils using a simple "loss-on-ignition" method. Commun. Soil Sci. Plant Anal., 20: 1675-1695. DOI: 10.1080/00103628909368175

9. Laiho, R., T. Penttila and J. Laine, 2004. Variation in soil nutrient concentration and bulk density within peatland forest sites. Silvia Fennica 38: 29-41. http://elektra.helsinki.fi/se/s/0037-5330/38/1/variatio.pdf 
11. Bray, R.H. and L.T. Kurtz, 1945. Determination of total, organic and available forms of phosphorus in soils. Soil Sci., 59: 39-45. http://scholar.google.com.pk/scholar?hl=en\&lr=\&q =info:2cWp5lf_TuMJ:scholar.google.com/\&output $=$ viewport\&pg $=1$

12. Driessen, P.M. and H. Suhardjo, 1977. Reclamation and use of Indonesian lowland peats and their effect on soil conditions. Proceedings of the 3rd ASEAN Soil Conference, (ASC'77), Kuala Lumpur, pp: 419-424.
13. Tie, Y.L. and H.S. Kueh, 1979. A review of lowland organic soils of Sarawak. Department of Agriculture, Technical Paper 4, Research Branch, Sarawak, Malaysia. http://library.wur.nl/isric/index2.html?url=http://lib rary.wur.nl/WebQuery/isric/16904 